\title{
Histology compared with chemical testing for urease for rapid detection of Helicobacter pylori in gastric biopsy specimens
}

\author{
A M Zaitoun
}

\begin{abstract}
Gastric biopsy specimens from 283 patients with ulcer and non-ulcer dyspepsia attending five gastroenterology clinics in the northern region of the United Arab Emirates (UAE) were tested by the agar gel test $(n=115)$ or the ultrarapid endoscopy room test $(n=168)$ for the presence of Helicobacter pylori urease. Results were compared with a histological technique using the Romanowsky type (Diff-3) stain for detecting $H$ pylori in both antral and body type gastric mucosa. A sensitivity of $94 \%$ and specificity of $100 \%$ using the agar gel test compared with $87 \%$ sensitivity and $99.3 \%$ specificity for the ultrarapid endoscopy room test. Grading of $H$ pylori in gastric biopsy specimens showed that the higher the histological grade, the more likely that the urease test would be positive.

Both forms of urease tests have high specificity for detecting $\boldsymbol{H}$ pylori in gastric biopsy specimens, although the urea agar test has a higher sensitivity than the ultra-rapid test. Low numbers of $H$ pylori in gastric biopsy specimens are the most important determinant of a false negative urease test.
\end{abstract}

(F Clin Pathol 1993;46:684-685)

A large body of evidence indicates that Helicobacter pylori is involved in the pathogenesis of chronic gastritis and perhaps in peptic ulcer disease. Several invasive and non-invasive techniques are currently used for detecting $H$ pylori infection. Of the invasive techniques, histology, culture, and the endoscopic biopsy urease test are the most widely used methods. ${ }^{1}$ The endoscopic biopsy urease test is a rapid diagnostic test based on the presence of the pre-formed enzyme in $H$ pylori in the specimens. Different forms of the test have been described including a CLO gel, ${ }^{2}$ a microtitre test, ${ }^{13}$ and urea broth and liquid forms. ${ }^{4-7}$ Each method has its advantages and disadvantages and the sensitivity has been reported to be in the region of $90 \%$.

This study aimed to assess the value of two endoscopic biopsy urease tests and to identify the possible factors responsible for their lower sensitivity than histological techniques which use the Sydney system for the classification of gastritis. $^{8}$

\section{Methods}

Five gastric biopsy specimens were taken routinely from 283 patients (89 females) with ulcer and non-ulcer dyspepsia from five hospitals.

The mean age of the patients was $37 \cdot 4$ years (range 12-100 years). One antral biopsy specimen was used for the urease agar gel test $(n=115)$ or for the ultra-rapid endoscopy room test $(n=168)$. For the former, the biopsy specimen was inserted into the gel of a urea agar slope (Biomèrieux, France). A positive result was indicated by a colour change to pink, usually after two hours at room temperature (up to nine hours). For the ultrarapid test, a large batch of $10 \%$ (weight/ volume) urea solution in deionised water at a $\mathrm{pH}$ of 6.8 was prepared and tested with an $H$ pylori strain isolated from an antral biopsy specimen before use. A $0.5 \mathrm{ml}$ aliquot of urea solution was placed in a capped tube and stored in a freezer at $-20^{\circ} \mathrm{C}$. Urea solution tubes were then dispatched to all five hospitals and only thawed before use. Two drops of a $1 \%$ phenol red solution were added to the test tube and an antral biopsy specimen was placed immediately into the urea solution. A positive result was recorded by the endoscopist if the colour changed from yellow to pink 1-15 minutes after insertion of the biopsy specimen. ${ }^{7}$

Four gastric biopsy specimens from the anterior and posterior wall of the antrum and corpus $^{8}$ were placed in $10 \%$ formol-saline. The specimens were then processed and sections $4 \mu \mathrm{m}$ thick were cut and stained by haematoxylin and eosin and Diff-3 stains. $^{9}$ The density of spiral bacteria was assessed blind according to the method of $\mathrm{Wyatt}^{10}$ in both antral and body type gastric biopsy specimens.

Each gastric biopsy was scored separately and the mean score of four biopsy specimens was used as the final score: $0=$ no curved bacteria seen; $1=$ one or two mucosa-associated bacteria seen; $2=H$ pylori in less than $50 \%$ of the gastric pits and surface area; $3=$ $H$ pylori in more than $50 \%$ of the pits or surface area; $4=$ dense $H$ pylori forming a carpet of bacteria.

Statistical evaluation was performed using the $\chi^{2}$ test with the Yates correction.

\section{Results}

Of the 115 patients with ulcer and non-ulcer dyspepsia, 14 had a negative urease gel test, six of which were found to have a false nega- 
Comparison of urease gel test and ultra-rapid endoscopy biopsy urease test with $H$ pylori grades obtained histologically from 283 patients with ulcer and non-ulcer dyspepsia

\begin{tabular}{|c|c|c|c|c|c|c|c|c|c|}
\hline & & \multicolumn{5}{|c|}{ H pylori grade } & \multirow[b]{2}{*}{ Total } & \multirow[b]{2}{*}{ Sensitivity \% } & \multirow{2}{*}{$\begin{array}{l}\text { Positive } \\
\text { predictive } \\
\text { value } \%\end{array}$} \\
\hline & & 0 & 1 & 2 & 3 & 4 & & & \\
\hline \multirow[t]{4}{*}{ Urease gel test } & & & & & & & & & \multirow{5}{*}{100} \\
\hline & Test negative & 8 & $t$ & 1 & 0 & 0 & \multirow{4}{*}{115} & * & \\
\hline & & 0 & & 1 & 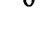 & 0 & & 94 & \\
\hline & $\begin{array}{l}\text { Test positive } \\
101\end{array}$ & 0 & $\begin{array}{l}\ddagger \\
6\end{array}$ & 56 & 26 & 13 & & & \\
\hline \multirow{4}{*}{ Ultra-rapid test } & & & & & & & & & \\
\hline & $\begin{array}{l}\text { Test negative } \\
36\end{array}$ & 14 & $\Delta$ & 8 & 1 & 0 & \multirow{3}{*}{168} & $\triangle$ & \multirow{3}{*}{$99 \cdot 3$} \\
\hline & & & & & & & & 87 & \\
\hline & $\begin{array}{l}\text { Test positive } \\
132\end{array}$ & 1 & 12 & 65 & 35 & 19 & & & \\
\hline
\end{tabular}

$\mathrm{p}$ value: $\nmid \mathrm{v} \neq, \Delta \mathrm{v} \backsim \mathrm{p}<0.0001 ;{ }^{\star} \mathrm{v} \Delta \mathrm{p}<0.05$

tive test compared with histology (table). The remaining 101 patients had positive urease gel tests which were confirmed histologically. $H$ pylori grades in gastric biopsy specimens stained by the Diff-3 stain are also shown in the table. Five out of six (83\%) patients with false negative urease tests had grade $1 H$ pylori compared with six out of $101(6 \%)$ with positive urease tests $(\mathrm{p}<0.0001)$. For $H$ pylori grade 2 , one patient had a false negative urease test compared with 56 patients with positive urease tests.

Thirty six of the 168 patients examined using the ultra-rapid urease test were found to be negative and 22 were false negative when compared with histology (table). Thirteen out of 22 had $H$ pylori grade 1 $(59 \%)$ in comparison with $9 \%$ with a positive ultra-rapid test $(p<0.0001)$. Only one patient out of 168 had a false positive ultrarapid test when histological examination did not show any organisms. The sensitivity of the urease gel test and the ultra-rapid urease test were $94 \%$ and $87 \%$, respectively ( $p<$ $0.05)$. The positive predictive values for both urease tests were similar (100v99.3\%).

\section{Discussion}

In this study, biopsy specimens were obtained following the recommendations of the Sydney system of classification of gastritis. ${ }^{8}$ Although the gastric antrum is the preferred site of biopsy for demonstration of $H$ pylori infection using the urease technique, histology, and culture, ${ }^{1}$ sampling both anterior and posterior walls of the body and antrum will effectively eliminate any false negative results caused by the patchiness of colonisation of gastric mucosa by $H$ pylori. The histological technique described in this study can be considered the gold standard for detecting $H$ pylori infection with which the urease test can be compared.

Most other authors have reported the sensitivity of the urease test to be comparable with the results found in this study. ${ }^{2-7} \mathrm{~A}$ $100 \%$ specificity of the urease test has been reported by other workers. ${ }^{3-7}$ The false positive result in one patient in the present study tested by the ultra-rapid test may be explained by the presence of other urease producing bacteria in the gastric biopsy specimen.

This study has shown that the higher the grade of $H$ pylori colonisation in the gastric biopsy specimen the more likely that the biopsy specimen will be positive on urease testing. There was a significant difference between false negative and true positive urease tests. This indicates that for patients with $H$ pylori grade 1 infection a significant number will have false negative tests.

Another possible cause of false negative urease tests is the complete absence of $H$ pylori in antral biopsy specimens due to the patchiness of organisms in patients who have intestinal metaplasia. ${ }^{11}$ Diff-3 stained smears have a sensitivity similar to the histological technique and can be performed and interpreted within minutes of endoscopic examination. ${ }^{9}$

It is concluded that both the urease gel and ultra-rapid urease tests have specificities of about $100 \%$ and sensitivities near $90 \%$. Grading of $H$ pylori in gastric biopsy specimens has shown that the higher the grade the more likely the biopsy urease test is to be positive and vice versa. The presence of a small number of $H$ pylori in the gastric biopsy specimen is the main determinant of a false negative urease test.

1 Schnell GA, Schubert TT. Usefulness of culture, histology and urease testing in the detection of Campylobacter pylori. Am $\mathcal{F}$ Gastroenterol 1989;84:133-7.

2 Marshall BJ, Warren JR, Francis GJ, Langton SR, Goodwin CS, Blincow ED. Rapid urease test in the management of Campylobacter pyloridis-associated gasmanagement of Campylobacter pyloridis-a.

3 Hazell SL, Borody TJ, Gal A, Lee A. Campylobacter pyloridis gastritis I: Detection of urease as a marker of pyloridis gastritis $\mathrm{I}$ : Detection of urease as a marker of 1987;82:292-6.

4 McNulty CAM, Dent JC, Uff JS, Gear MWL, Wilkinson SP. Detection of Campylobacter pylori by the biopsy urease test: An assessment in 1445 patients. Gut 1989;30:1058-2.

5 Das SS, Bain LA, Karim QN, Coelho LG, Baron JH. Rapid diagnosis of Campylobacter pyloridis infection $\dot{f}$ Clin Pathol 1987;40:701-2.

6 Vaira D, Holton J, Cairns S, Falzon M, Salmon P. Four hour rapid urease test (RLT) for detecting Campylobacter pylori: is it reliable enough to start treatment? $f$ Clin Pathol 1988;41:355-6.

7 Thillainayagam AV, Arvind AS, Cook RS, Harrison TG, Tabagchali S, Farthing MJG. Diagnostic efficiency of an ultrarapid endoscopy room test for Helicobacter pylori. ultrarapid endoscopy

8 Price AB. The Sydney System: Histological division. $f$ Gastroenterol Hepatol 1991;6:209-22.

9 Zaitoun AM. Use of Romanowsky type (Diff-3) stain for detecting helicobacter pylori in smears and tissue sections. F Clin Pathol 1992;45:448-9.

10 Wyatt JI. The distribution of Campylobacter pylori in the gastric mucosa in chronic gastritis and the effect of cimetidine. In: Takemoto $T$, Kawai $K$, Shimoyama $T$, eds. Campylobacter pylori and gastroduodenal diseases. Vol. 2. Proceedings of the 2nd Tokyo International Symposium on Campylobacter pylori. Tokyo: Taisho Pharmaceutical, 1990;152-8.

11 Marshall BJ, McGechie DB, Rogers PA, Glancy RJ. Pyloric Campylobacter infection and gastroduodenal disease. Med $\mathcal{F}$ Aust 1985;142:439-44. 